\title{
Yttrium ferrite based circularly polarized triangular patch array antenna
}

\author{
Dheeraj Kumar and P.K.S. Pourush ${ }^{1}$ \\ Department of Applied Physics, Delhi Technological University, New Delhi 110 042, India. \\ ${ }^{1}$ Microwave \& Antenna System Lab, Deptartment of Physics, Agra College, Agra-282 002, India \\ kdheeraj_7@yahoo.co.in
}

\begin{abstract}
In this communication, the circularly polarized properties of $4 \times 4$ element planar microstrip equilateral triangular array antenna (METAA) printed on Yttrium ferrite substrate are presented. Two hands of circular polarization are generated when the magnetic bias is applied parallel to the wave propagation in the antenna geometry. The values of antenna parameters of array geometry as radiation conductance, radiation efficiency and bandwidth are investigated for biased and unbiased case. The analysis is based on cavity model.
\end{abstract}

Keywords: Antenna, ferrite, microstrip array, circular polarization, Yttrium, radiation.

Introduction

One of the most important applications microstrip antennas is generation of circular polarization, which is widely required for many satellite and radar communication systems. This circular polarization is obtained when two orthogonal modes are excited with equal amplitude and in phase quadrature. For conventional patch antennas, this requires a minimum two feeds for the fundamental mode and usually four feeds at the correct angular spacing for higher modes. However, single feed microstrip antennas for circular polarization are usually achieved by truncating patch corners of square patch, using nearly square or nearly circular patches, inserting a pair of symmetric perturbation elements at a boundary of a circular patch (Roy \& Vandon, 1992; Chen \& Wong, 1999; Haung, 1999; Iwasaki \& Chiva, 1999). A determination of optimum feed location is essentially required. For circular polarization on ferrites, the degenerate orthogonal modes are easily generated with an application of a dc magnetic bias field normal to the ferrite and patch. Due to the magnetic anisotropy of the ferrite, the cavity field components are degenerate with the $90^{\circ}$ phase difference generating circular polarization. Hence, a single feed point together with magnetic bias produces circular polarization not only the fundamental mode also for the higher order modes, thereby saving considerably on feed complexity. This is a considerable advantage over dielectric based antennas where much more complex networks are necessary, usually two or four correctly phased probes (Naveen Kumar Saxena et al., 2010).

\section{Theory}

By the application of DC magnetic bias parallel to wave propagation in the ferrite substrate, the permeability character of the ferrite become a tensor quantity, which consist of frequency dependent parameters ' $\mu$ ' and ' $k$ ' defined as:

$$
\begin{aligned}
& \mu=1+\frac{\omega_{0} \omega_{m}}{\left(\omega_{0}^{2}-\omega_{m}{ }^{2}\right)} \\
& k=\frac{\omega \omega_{m}}{\left(\omega_{0}{ }^{2}-\omega^{2}\right)}
\end{aligned}
$$

$$
\text { Where, } \omega_{0}=\gamma H_{0} \text { and } \omega_{m}=\gamma 4 \pi M_{s}
$$

Here $4 \pi M_{s}$ is the saturation magnetization, $H_{0}$ is applied magnetic field strength, $\gamma$ is the gyromagnetic ratio $(=2.8 \mathrm{MHz} / \mathrm{Oe})$ and $\omega_{0}$ and $\omega_{\mathrm{m}}$ are the precession and forced precession frequencies, respectively.

Now the effective permeability ' $\mu_{\text {eff }}$ ' and propagation constant ' $\mathrm{K}$ ' for this case are defined as:

$$
\begin{gathered}
\mu_{\text {eff }}=\mu \pm k \\
\left(\frac{K_{ \pm}}{K_{o}}\right)^{2}=\mu \pm k
\end{gathered}
$$

Here, the modes with propagation constants $\mathrm{K}_{+}$and $\mathrm{K}$ are called right hand circularly polarized (RHCP) and left hand circularly polarized (LHCP) modes respectively. Since, under applied field, an incident linearly polarized wave is resolved in two counter-rotating circularly polarized waves, each of which does propagate as a mode. The explicit dependence of the propagation constant on basic parameters is obtained on substituting for $\mu$ and $k$ and finally the relation is of the form as:

$$
\frac{K_{ \pm}}{K}=\left(\frac{\omega_{0}+\omega_{m} \mp \omega}{\omega_{0} \mp \omega}\right)^{\frac{1}{2}}
$$

The behavior of RHCP and LHCP modes can be qualitatively understood as in the presence of applied magnetic field; the magnetic dipoles in the ferrite medium
Dheeraj Kumar \& Pourush Indian J.Sci.Technol. 
Indian Journal of Science and Technology

Fig. 2. Variation in field pattern of METAA with angle of incidence for unbiased case

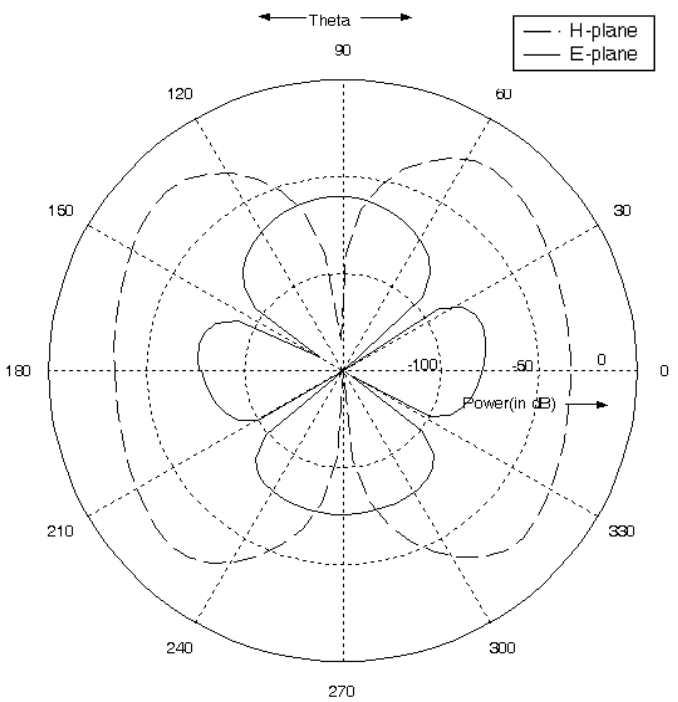

Fig. 3. Variation in field pattern of METAA with angle of incidence for biased case in RHCP

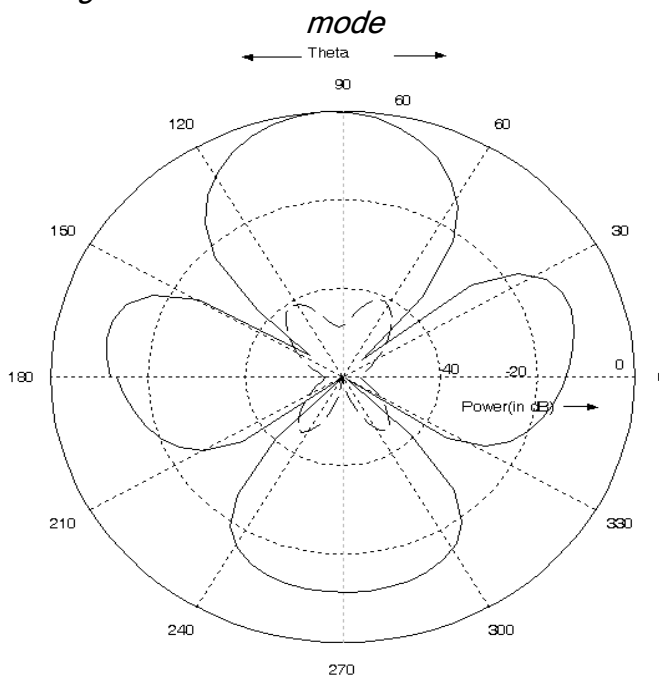

Fig. 4. Variation in field pattern of METAA with angle of incidence for biased case in LHCP mode

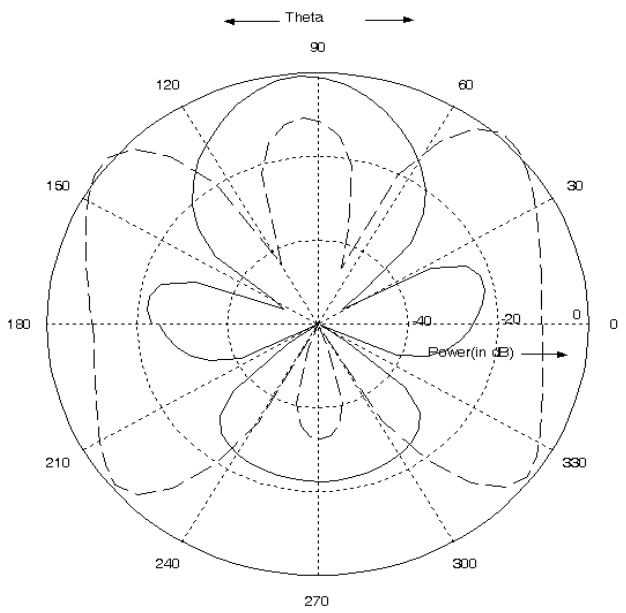

Research communication

CIndian Society for Education and Environment (iSee)

Vol. 3 No. 4 (Apr. 2010)

ISSN: 0974- 6846

Fig. 1. Geometry of triangular array microstrip patch antenna

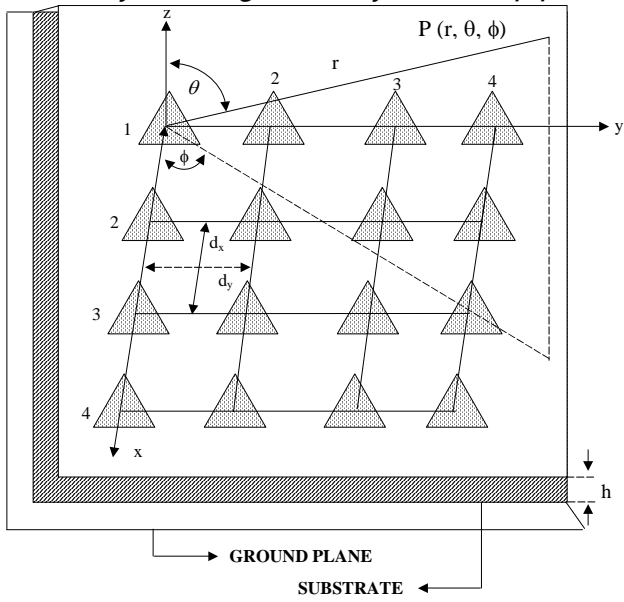

Fig. 5. Variation in Efficiency of METAA with magnetic field

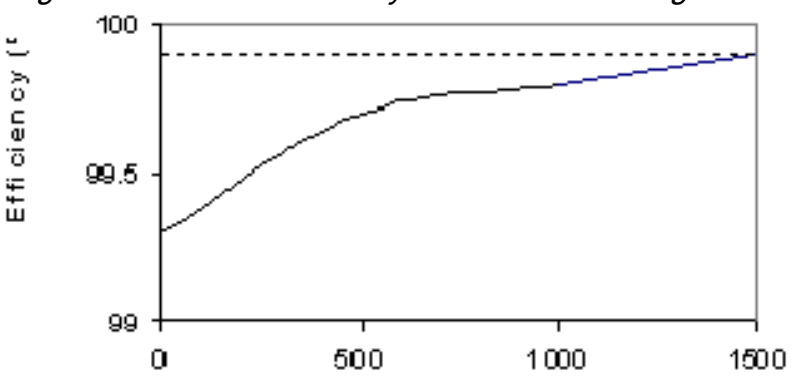

Magnetic field ( Oe)

Fiq. 6. Variation in Bandwidth of METAA with maqnetic field

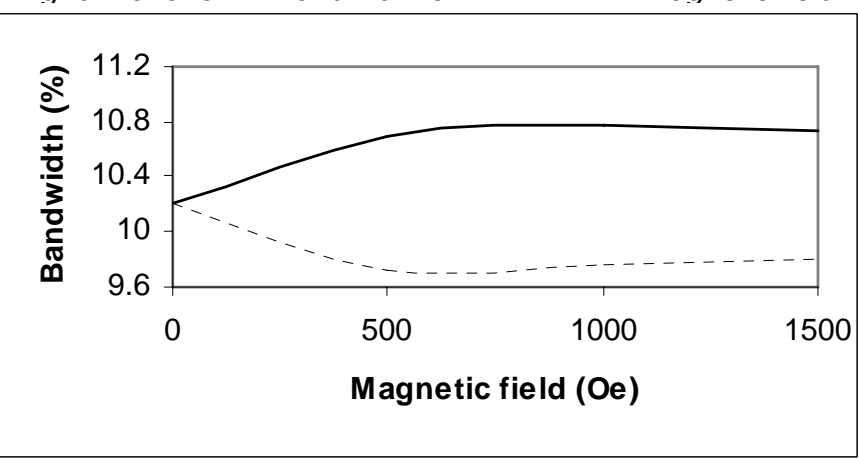

Fig. 7. Variation in radiation conductance of METAA with magnetic field

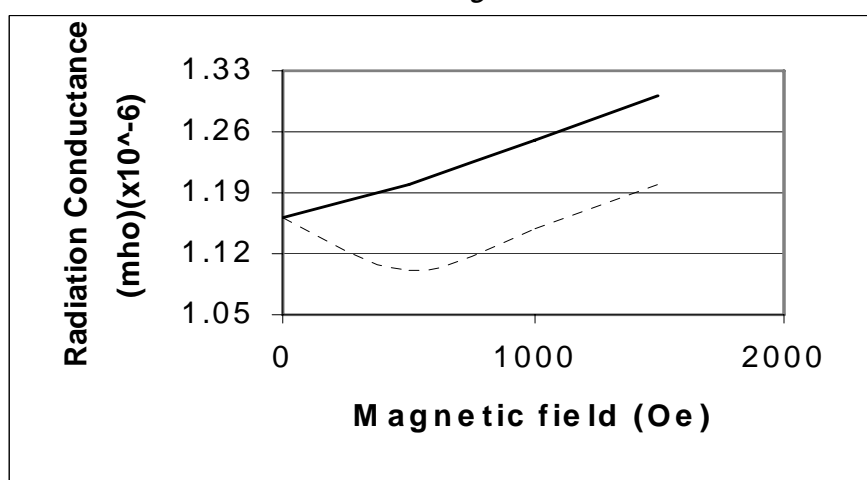

"Antenna" http://www.indjst.org
Dheeraj Kumar \& Pourush Indian J.Sci.Technol. 
execute a clockwise precession about the field with the precession along the applied magnetic field of RHCP wave propagation. The applied magnetic field also rotates in the clockwise direction and hence enhances the amplitude of precession of the dipoles. Therefore, the RHCP mode interacts strongly with the medium and, specially includes resonance when the two frequencies are equal, i.e. when $\omega=\omega_{0}$. On the other hand, the tendency of the LHCP mode is obviously to oppose the precession of dipoles. Hence, this mode interacts rather weakly with the medium (Soda, 1981).

\section{Antenna configuration}

The geometry and co-ordinates system of METAA is shown in Fig. 1. It consists 16 equilateral triangular patch elements of side 'a' printed on ferrite of thickness $(h)=$ $1.27 \mathrm{~mm}, \mathrm{a}=1.25 \mathrm{~cm}$, substrate permittivity $\left(\varepsilon_{\mathrm{r}}\right)=16$, loss tangent $(\tan \delta)=0.0005$, saturation magnetization $\left(4 \sqcap \mathrm{M}_{s}\right)$ $=1800$ Gauss. Element separation $\left(d_{x}\right.$ and $\left.d_{y}\right)$ and progressive phase excitation difference $\left(\beta_{x}\right.$ and $\left.\beta_{y}\right)$ between the the elements is $1.0 \mathrm{~mm}$ and $n / 4$ respectively. The resonant frequency $\left(\mathrm{f}_{\mathrm{r}}\right)$ of antenna is $4 \mathrm{GHz}$, and the external bias field $\left(\mathrm{H}_{0}\right)$ applied over it is $1500 \mathrm{Oe}$.

\section{Results and discussion}

The field patterns for the antenna are computed and plotted under unbiased and biased case (RHCP mode and LHCP mode) for given parameters in two different planes (i.e. E-plane and $\mathrm{H}$-plane). The patterns are shown in Fig. 2-4 for unbiased case RHCP mode and LHCP mode respectively. It is observed from the figures that patterns of array antenna provide limited number of secondary lobes. It is found that on application of biasing, the position of the main beam and the secondary beams are shifted. Obviously METAA patterns scanned because of application of external biasing, which is in similar fashion as reported by Batchelor and Langley (1997).

The antenna parameters of METAA radiation conductance, efficiency and bandwidth are computed and plotted for RHCP and LHCP mode with respect to external magnetic bias field using the above expressions taking the same input parameters. The variation in radiation conductance, efficiency and bandwidth of the array are shown in Fig. 5, 6 and 7 respectively. The results are in consistent with that reported by Pozar (1992).

\section{Conclusion}

The radiation characteristics of METAA under biased and unbiased cases have been examined through the use of a cavity model analysis. The physical effects such as dielectric loss and conductor loss are included. The effects of changing the bias field intensity on radiation conductance, efficiency and bandwidth, for the RHCP and LHCP mode have been investigated. It is found that there is a significant change in the values of antenna parameters by the variation in external magnetic field. It is observed that beam scanning, lobe enhancement or reducing, bandwidth adjustment and pattern reshaping can be obtained by varying the bias. The device has low profile and the application of the magnetic field has to be over a small area for generating circular polarization. This analysis has potential use in phased arrays, miniaturization of antenna systems, which are applicable in space and cellular communication.

\section{References}

1. Bahl IJ and Bhartia P (1980) Microstrip Antenna. Artech House. pp: 139-179.

2. Batchelor JC and Langley RJ (1997) Beam scanning using microstrip line on biased Ferrite. Electronic Lett. $33,8$.

3. Chen HM and Wong KL (1999) On the circular polarization operation of annular ring microstrip antenna. IEEE Trans. Antennas Propagation. 47(8), 1289-1292.

4. Haung CY (1999) Design for an aperture coupled compact circularly polarized microstrip antenna. IEE Proc. Microwaves. Antennas Propagation. 146(1), 1316.

5. Iwasaki H and Chiva N (1999) Circular polarized back to back microstrip antenna with an ominidirectional pattern. IEE Proc. Microwave Antennas Propagation. 146(4), 277-281.

6. Naveen Kumar Saxena, Moh. Ayub Khan, Nitendar Kumar and P.K.S. Pourush (2010) LiTiMg ferrite substrate for circular array of circular patches under external magnetic field. Indian J.Sci.Technol. 3 (3), 299-302. Domain site: http://www.indjst.org.

7. Pozar DM (1992) Radiation and scattering characteristics of microstrip antenna on normally biased Ferrite substrate. IEEE Trans. A\&P, AP-40, 1084-1092.

8. Roy JS and Vandon P (1992) Circularly polarized for field of an axially magnetized circular Ferrite microstrip antenna. Microwave Opt. Tech. Letts. 5, 228-230.

9. Soda MS Srivastava NC (1981) Microwave propagation in Ferrimagnetics. Plenum Press, New York and London.

\section{"Antenna"} http://www.indjst.org
Dheeraj Kumar \& Pourush Indian J.Sci.Technol. 